\title{
Morfología de estructuras parasitarias de Rangelia vitalii en muestras de perros naturalmente infectados
}

\author{
Morphology of Rangelia vitalii parasitic stages in samples from \\ naturally infected dogs
}

\section{Sánchez RO ${ }^{1^{*}}$, Moré $\mathrm{G}^{2,3}$, Eiras $\mathrm{D}^{3,4}$}

${ }^{1}$ Laboratorio Mesopotámico de Diagnóstico Veterinario, Concordia, Entre Ríos; ${ }^{2}$ Consejo Nacional de Investigaciones Científicas y Técnicas (CONICET); ${ }^{2}$ Laboratorio de Inmunoparasitología, Facultad de Ciencias Veterinarias, Universidad Nacional de La Plata; ${ }^{4}$ Laboratorio DIAP (Diagnóstico en Animales Pequeños), Banfield, Buenos Aires, Argentina.

Correo electrónico del autor: ricardosanchez74@gmail.com

\begin{abstract}
Resumen: La rangeliosis es una hemoparasitosis causada por Rangelia vitalii y transmitida por la garrapata Amblyomma aureolatum, que afecta a perros y otros cánidos silvestres. La enfermedad ha sido diagnosticada en Brasil desde principios del siglo XX, en Uruguay y, recientemente, en Argentina. El parásito es un protozoo intracelular Apicomplexa que está emparentado filogenéticamente con $\mathrm{Ba}$ besia spp., aunque presenta diferencias en su ciclo parasitario. Se han observado formas parasitarias dentro de eritrocitos, monocitos, neutrófilos y en el endotelio capilar de diversos órganos, como así también libres en plasma. El presente trabajo describe la morfología de cuatro formas parasitarias (o estadios), observadas en sangre de caninos naturalmente infectados por Rangelia vitalii en Argentina.
\end{abstract}

Palabras clave: Rangelia vitalii, morfología, diagnóstico

\begin{abstract}
Rangeliosis is a tick-borne disease affecting domestic and wild canids caused by Rangelia vitalii and transmitted by the tick Amblyomma aureolatum. The disease has been diagnosed in southern Brazil since early XX century, in Uruguay, and, recently, in Argentina. The parasite is an intracellular Apicomplexan protozoon phylogenetically close to Babesia spp. However, it shows differences in the life cycle. Parasitic stages have been observed in monocytes, erythrocytes and neutrophils, and in endothelial cells from several organs, as well as free in plasma. The present study describes the morphology of four different parasitic stages observed in blood samples from naturally infected dogs in Argentina.
\end{abstract}

Key words: Rangelia vitalii, morphology, diagnosis 


\section{Introducción}

La rangeliosis canina es una hemoparasitosis transmitida por garrapatas que afecta a los cánidos y es provocada por un protozoo del phylum Apicomplexa y del orden Piroplasmida denominado Rangelia vitalii. La enfermedad fue diagnosticada en Brasil como causada por un piroplasma, Piroplasma vitalii, a principios del siglo XX (Pestana, 1910). Inicialmente se clasificó al agente como perteneciente al género Babesia, pero actualmente se encuentra clasificado en un género diferente, con diferencias morfológicas, biológicas y moleculares que lo separan de los piroplasmas pertenecientes a los géneros Babesia y Theileria. En género fue denominado Rangelia como fuera propuesto en 1914 (Carini \& Maciel, 1914; Soares et al., 2011). Los signos clínicos más frecuentes pueden incluir anorexia, apatía, anemia, ictericia, hemoglobinuria, hemorragias subcutáneas, hepatomegalia, esplenomegalia, linfoadenomegalia, diarrea con o sin sangre y sangrado espontáneo de orejas, nariz y otras partes del cuerpo (Da Silva et al., 2011, 2012; Fighera et al., 2010; França et al., 2010; Lorreti \& Barros, 2005; Soares, 2014).

Hasta hace poco tiempo se creía que la garrapata marrón del perro (Rhipicephalus sanguineus) estaba implicada en la transmisión de la enfermedad. Sin embargo, estudios recientes han permitido descartarla y demostrar que Amblyomma aureolatum es el principal vector capaz de transmitir la enfermedad, de manera transovárica y transestadial (Soares, 2014).

El principal método de diagnóstico es la observación de estadios del protozoo en muestras de sangre, médula ósea o tejidos de los cánidos infectados. Se reporta frecuentemente la presencia de estadios extraeritrocitarios libres en el plasma o dentro de células sanguíneas (eritrocitos, neutrófilos y monocitos) y células endoteliales de diversos órganos, particularidad que justifica en gran medida la separación taxonómica de este piroplasma de aquellos pertenecientes al género Babesia (Lorreti \& Barros 2005, Soares et al., 2011).

Luego de la inoculación experimental del parásito en caninos, a partir del quinto día post infección (DPI) pueden comenzar a observarse eritrocitos infectados en sangre capilar. La cantidad de eritrocitos infectados alcanza su pico alrededor de los 9-11 DPI. Entre los 17 y los $21 \mathrm{DPI}$, los parásitos se observan mayormente dentro de leucocitos; hacia los $30 \mathrm{DPI}$ ya no suelen detectarse en sangre periférica (Da Silva et al., 2011). Durante la infección natural, los eritrocitos infectados son detectables en sangre periférica después de las 2 semanas posinfección, en coincidencia con la trombocitopenia (Soares, 2014).

Esta enfermedad es endémica en el sur y sureste de Brasil, afectando a cánidos domésticos y sil- vestres y ha sido también diagnosticada en el norte de Uruguay (Fredo et al., 2015; Sarasúa \& Donati, 1976; Soares et al., 2015). La dispersión de la enfermedad puede atribuirse a la infección en cánidos silvestres, ya que la garrapata transmisora se encuentra asociada a ambientes periurbanos o rurales, aunque se considera posible la colonización de ambientes urbanos (Loretti \& Barros, 2005; Soares, 2014).

En nuestro país, la enfermedad ha sido diagnosticada en las provincias de Misiones y Entre Ríos (Eiras et al., 2014; Sánchez et al., 2014). Existen registros de A. aureolatum en las provincias de Chaco, Misiones, Entre Ríos (Guglielmone et al., 2002) y, recientemente, en Buenos Aires y Santa Fe (Colombo et al., 2016), por lo que la distribución de la rangeliosis canina podría ser mayor a la actualmente informada.

El objetivo de este trabajo fue describir morfológicamente las formas parasitarias halladas en sangre de perros naturalmente infectados con $R$. vitalii, en la provincia de Entre Ríos.

\section{Descripción metodológica}

Las muestras de sangre incluidas en el estudio correspondieron a 17 perros de la provincia de Entre Ríos con manifestaciones clínicas compatibles con rangeliosis y con diagnóstico confirmado en el Laboratorio Mesopotámico de Diagnóstico Veterinario en Concordia, Entre Ríos. La confirmación etiológica $(R$. vitalii) se realizó mediante estudios microscópicos (confirmación parasitológica) de todas las muestras y en 3 casos se realizó PCR anidada RFLP y PCR anidada y secuenciación como se describió anteriormente (Eiras et al., 2014). Los extendidos de sangre periférica y/o capilar (punción del pabellón auricular) fueron coloreados con Tinción 15 (Biopur®) o May Grünwald-Giemsa. Los frotis en los que se observaron merozoítos de piroplasmas dentro de leucocitos circulantes fueron considerados positivos para $R$. vitalii, desde el punto de vista parasitológico. Las mediciones fueron realizadas a 1000 aumentos bajo aceite de inmersión, mediante microscopio dotado de regla micrométrica.

Se observaron merozoítos de $R$. vitalii con diferentes formas, extra e intracelulares. Estas se clasificaron en cuatro grupos: merozoítos libres tipo 1 y tipo 2, merozoítos intraleucocitarios y merozoítos intraeritrocitarios. Las medidas halladas para las diferentes estructuras se resumen en la Tabla 1.

Merozoítos libres tipo 1: estas formas fueron observadas principalmente en muestras de sangre capilar y se presentaron en forma solitaria o en grupos. Poseían generalmente forma piriforme, aunque se observaron algunos redondos, ovales e, incluso, irregulares. Presentaron citoplasma levemente basófilo, 


\section{Sánchez RO et al.}

Tabla 1. Medidas de las diferentes formas parasitarias observadas en frotis sanguíneos de perros infectados naturalmente con Rangelia vitalii.

\begin{tabular}{|c|c|c|c|c|c|c|c|c|}
\hline & \multicolumn{2}{|c|}{$\begin{array}{c}\text { merozoítos libres } \\
\text { tipo1 }\end{array}$} & \multicolumn{2}{|c|}{$\begin{array}{c}\text { merozoítos libres } \\
\text { tipo } 2\end{array}$} & \multicolumn{2}{|c|}{$\begin{array}{c}\text { merozoítos } \\
\text { intraleucocitarios }\end{array}$} & \multicolumn{2}{|c|}{$\begin{array}{c}\text { merozoítos } \\
\text { intraeritrocitarios }\end{array}$} \\
\hline & largo $(\mu \mathrm{m})$ & $\begin{array}{c}\text { ancho } \\
(\mu \mathrm{m})\end{array}$ & $\begin{array}{c}\text { largo } \\
(\mu \mathrm{m})\end{array}$ & $\begin{array}{c}\text { ancho } \\
(\mu \mathrm{m})\end{array}$ & $\begin{array}{l}\text { largo } \\
(\mu \mathrm{m})\end{array}$ & $\begin{array}{c}\text { ancho } \\
(\mu \mathrm{m})\end{array}$ & $\begin{array}{l}\text { largo } \\
(\mu \mathrm{m})\end{array}$ & $\begin{array}{c}\text { ancho } \\
(\mu \mathrm{m})\end{array}$ \\
\hline Promedio & 3,6 & 2,6 & 4,0 & 2,8 & 2,4 & 1,8 & 3,6 & 2,3 \\
\hline Moda & 4,0 & 3,0 & 3,5 & 3,0 & 3,0 & 2,0 & 3,0 & 2,0 \\
\hline DesvEst & 0,55 & 0,42 & 0,67 & 0,42 & 0,52 & 0,32 & 0,79 & 0,39 \\
\hline Rango & $2,5-5$ & $2-3,5$ & $3-5,5$ & $2,5-4$ & $1,5-3$ & $1,5-2,5$ & $2,5-6$ & $1,5-3$ \\
\hline$n$ & 57 & 57 & 26 & 26 & 33 & 33 & 22 & 22 \\
\hline
\end{tabular}

una estructura redonda u ovalada más basófila que el citoplasma de 0,5 a $1 \mu \mathrm{m}$ de diámetro, generalmente situada en el extremo más delgado y un núcleo redondeado u oval de color magenta o basófilo de 1 a $2 \mu \mathrm{m}$ de diámetro que abarcaba, frecuentemente, la mitad o todo el ancho del parásito. Las formas irregulares muchas veces presentaron estructuras internas basófilas dobles (Figuras 1 y 2 ).

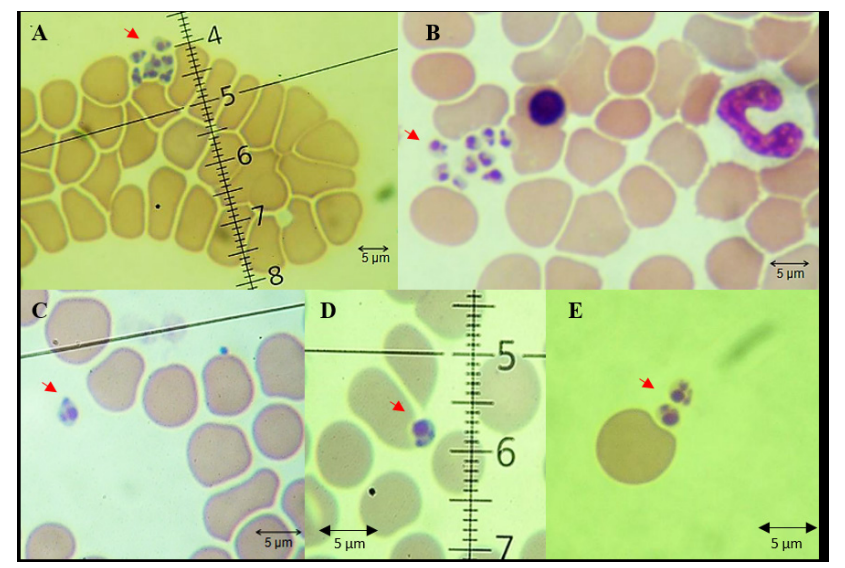

Figura $1 \mathrm{~A}$-E. Merozoítos libres tipo 1 de $R$. vitalii (flechas rojas) en frotis de sangre de caninos.

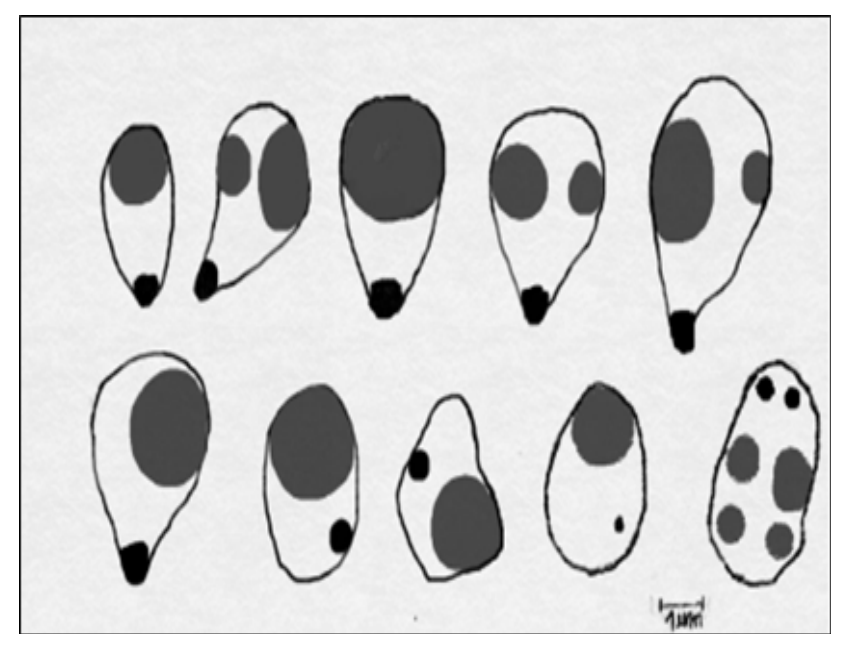

Figura 2: Esquema de los merozoítos libres tipo 1.
Merozoítos libres tipo 2: estas estructuras, también extracelulares, presentaron formas globosas, ahusadas o amorfas, con un núcleo redondo u oval de 0,5 a $1 \mu \mathrm{m}$ de diámetro y citoplasma levemente basófilo. Estas formas midieron, en promedio, $4 \mu \mathrm{m}$ de largo por 2,8 $\mu \mathrm{m}$ de ancho. Se observaron solas o agrupadas e incluso, en ocasiones, rodeadas por una fina membrana formando una vesícula que englobaba a varias formas parasitarias (Figuras 3 y 4 ).

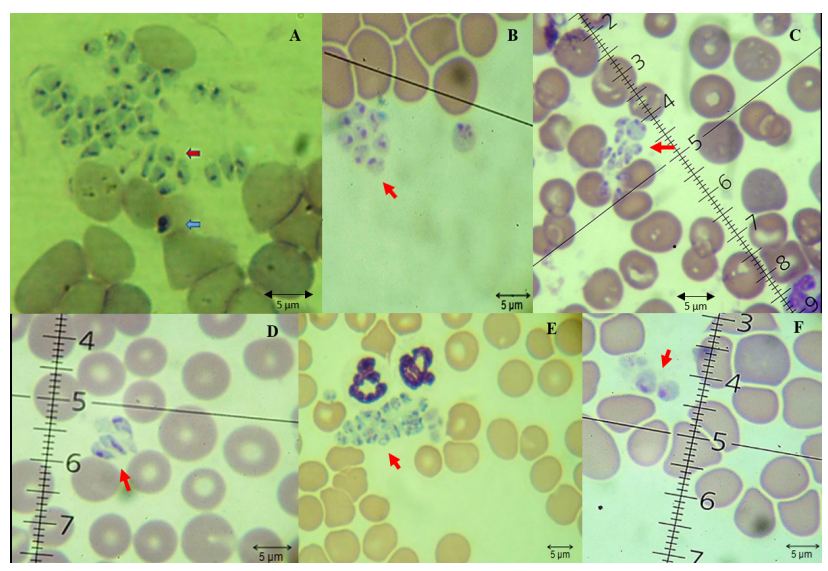

Figura 3 A-F. Merozoítos de R. vitalii libres en plasma: merozoítos libres tipo 1 (flecha azul) y merozoítos libres tipo 2 (flechas rojas).

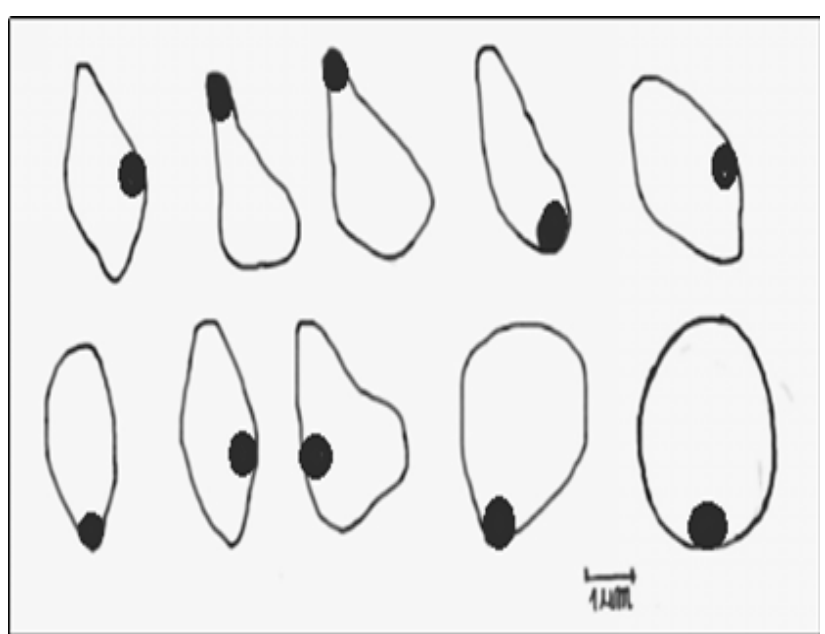

Figura 4. Esquema de los merozoítos libres tipo 2. 
Merozoítos intraleucocitarios: se observaron dentro de monocitos y neutrófilos, eran piriformes a ovalados, de 2,4 $\mu \mathrm{m}$ de largo por 1,8 $\mu \mathrm{m}$ de ancho en promedio, con un núcleo basófilo redondeado de 0,5 a $1 \mu \mathrm{m}$, ubicado en el extremo apical, y un citoplasma generalmente acidófilo. Dentro de los monocitos se observaron una o más vacuolas con varios parásitos en su interior, o formas libres en el citosol, mientras que en los neutrófilos solo se observaron formas libres. Dentro de los monocitos también pudieron observarse formas similares a los merozoítos tipo 1 (Figuras 5 y 6 ).

Merozoítos intraeritrocitarios: los merozoítos observados dentro de glóbulos rojos presentaron forma piriforme, redondeada, hasta amorfa. Los merozoítos presentaron citoplasma levemente basófilo, con un promedio de 3,6 $\mu \mathrm{m}$ de largo por $2,3 \mu \mathrm{m}$ de ancho y un pequeño núcleo basófilo de 0,5 a $1 \mu \mathrm{m}$ de diámetro. No se observaron más de 2 merozoítos por eritrocito (Figuras 7 y 8 ).

\section{Discusión y conclusiones}

La rangeliosis canina es una enfermedad que en la actualidad se ha diagnosticado desde el sur de Brasil hasta Uruguay y el noreste argentino. Si bien se ha podido reproducir la infección en forma experimental, no se ha estudiado en profundidad la morfología de los diferentes estadios del ciclo biológico en infecciones naturales. Este protozoo realiza una etapa de merogonia dentro de células como monocitos, neutrófilos y células endoteliales, como sucede en otros hemoparásitos (Plasmodium spp., Theileria spp., Cytauxzoon spp. etc.). Sin embargo, los estudios filogenéticos han demostrado una estrecha relación entre $R$. vitalii y los piroplasmas intraeritrocitarios del género Babesia (Eiras et al., 2014; Soares, 2011; Soares et al., 2014). De acuerdo con el gen estudiado y el método utilizado para el análisis filogenético, $R$. vitalii se ubicó dentro del clado de Babesia spp. o en su base. Se postula que $R$. vitalii constituye un taxón diferente dentro de las especies de piroplasmas que afectan a caninos, aunque se necesitan más estudios para definir el posicionamiento filogenético de este parásito (Franca et al., 2014).

Existen datos morfométricos de $R$. vitalii que fueron aportados por Da Silva et al., (2011), quienes realizaron mediciones de 3 estadios parasitarios. Sin embargo, ellos no mencionan diferencias entre los parásitos observados intra o extracelularmente ni aportan detalles morfológicos. Las medidas promedio de parásitos extracelulares hallados en plasma por estos autores fueron de 3,94 $\pm 0,67 \mu \mathrm{m}$ de largo por 2,23 \pm $0,42 \mu \mathrm{m}$ de ancho. El tamaño del núcleo fue de $0,97 \pm$ $0,17 \mu \mathrm{m}$ de largo por $0,84 \pm 0,11 \mu \mathrm{m}$ de ancho. Estos datos coinciden parcialmente con los del presente estudio, ya que en nuestros hallazgos los merozoítos

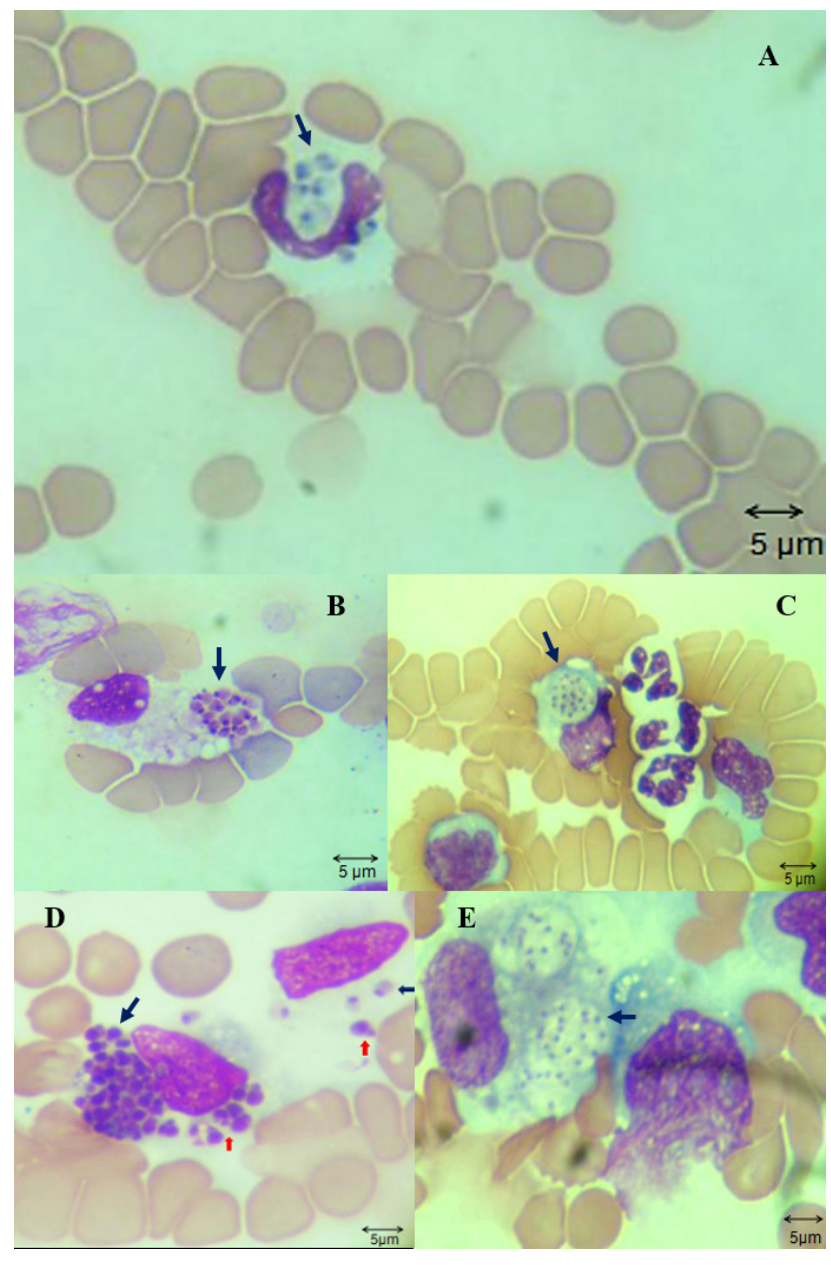

Figura 5 A-E. Merozoítos intraleucocitarios en neutrófilo $(A)$ y en monocito (B-E) indicados con flechas azules. También se observan merozoítos libres tipo 1 (flechas rojas) (D).

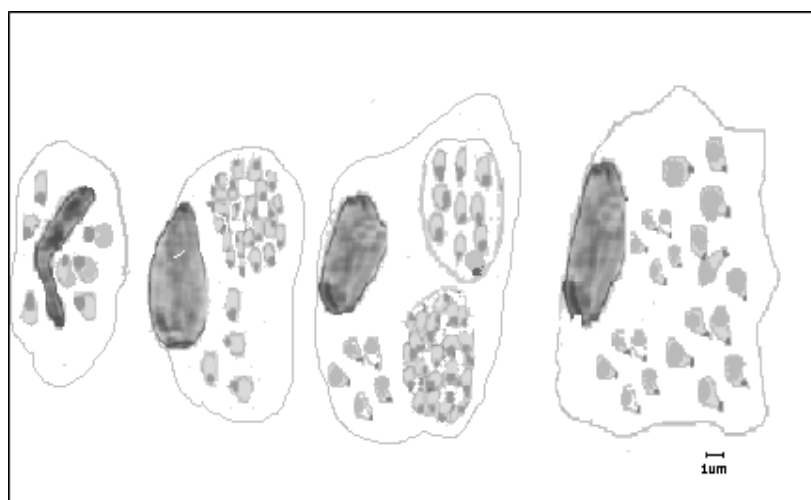

Figura 6. Esquema de los merozoítos intraleucocitarios.

fueron algo más anchos. Los merozoítos libres tipo 1 presentaron como característica una estructura basófila en la parte más angosta y la presencia de otra u otras estructuras basófilas o magenta de mayor tamaño que podrían representar al núcleo en etapas de división. Loretti \& Barros (2005) realizaron estudios de formas parasitarias encontradas dentro de células endoteliales mediante microscopía electrónica. Ellos describieron organismos redondos $u$ ovalados con 


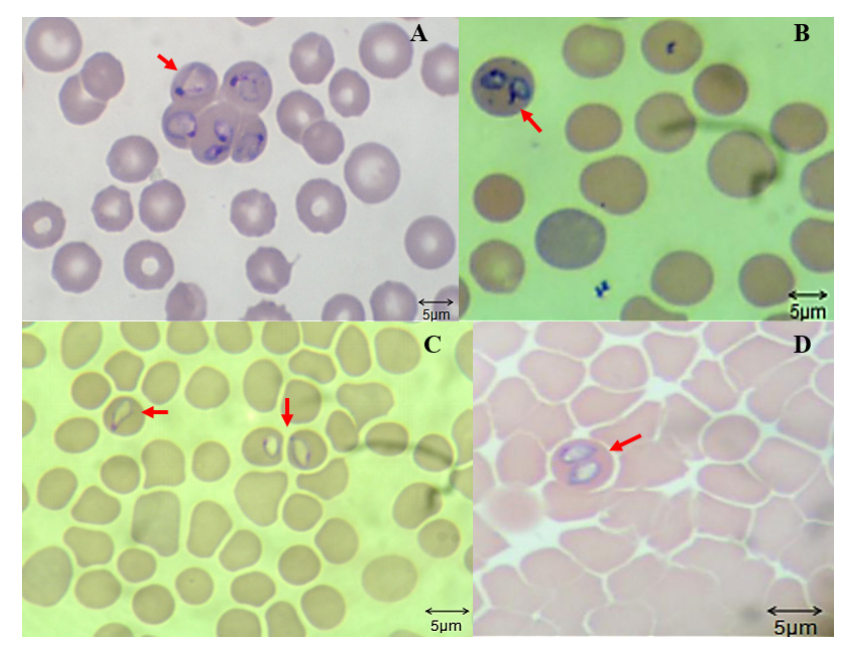

Figura 7 A-D. Merozoítos intraeritrocitarios de R. vitalii (flechas rojas).

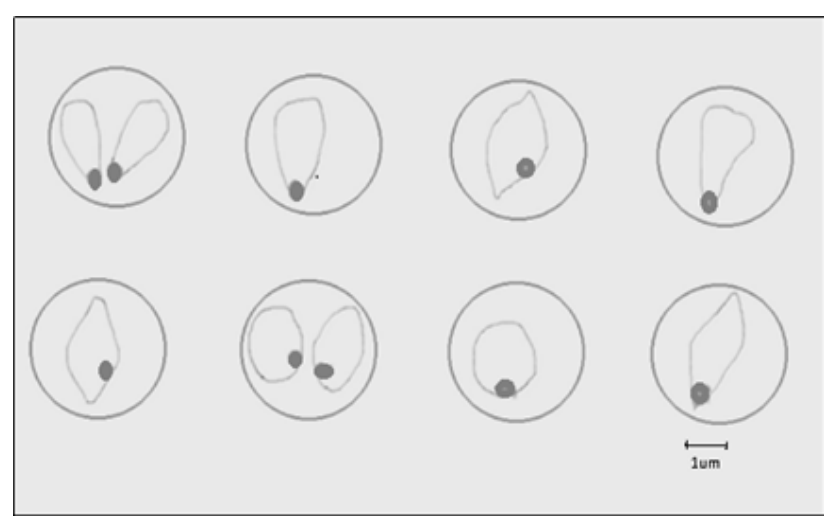

Figura 8. Esquema de los merozoítos intraeritrocitarios.

núcleo excéntrico electrondenso, rodeados por una membrana parasitófora trilaminar y un complejo apical sin conoide. Además, observaron inclusiones cristalinas de forma variable en el citosol de los organismos situados en el endotelio de los capilares intersticiales del riñón. Lamentablemente, no existen estudios de microscopía electrónica realizados sobre otras formas parasitarias identificadas, ya sea dentro de leucocitos, eritrocitos o las halladas libres en plasma.

La longitud de los parásitos (dentro de los eritrocitos) medidos en este trabajo es similar a la observada por Da Silva et al. (2011), quienes observaron, en promedio, 3,34 $\pm 0,54 \mu \mathrm{m}$ de largo por 2,09 $\pm 0,34 \mu \mathrm{m}$ de ancho. En promedio, el núcleo midió 1,07 $\pm 0,20$ $\mu \mathrm{m}$ de largo por $0,87 \pm 0,15 \mu \mathrm{m}$ de ancho.

Las medidas de las formas parasitarias observadas dentro de los leucocitos por Da Silva et al. (2011) fueron de 2,97 $\pm 0,68 \mu \mathrm{m}$ de longitud por 1,94 $\pm 0,26 \mu \mathrm{m}$ de ancho. El promedio de longitud de los núcleos fue $1,17 \pm 0,34 \mu \mathrm{m}$ de largo por $0,85 \pm 0,23$ $\mu \mathrm{m}$ de ancho. En este trabajo, el promedio hallado fue levemente menor, $2,4 \pm 0,54 \mu \mathrm{m}$ de largo por 1,8 \pm $0,34 \mu \mathrm{m}$ de ancho. Estas formas parásitas parecieran ser similares a las descriptas en estudios histopato- lógicos dentro de células endoteliales (Fighera et al., 2010; Loretti \& Barros, 2005). En ocasiones, también fueron observadas estructuras parasitarias similares a los merozoítos tipo 1 , aunque de no más de $3 \mu \mathrm{m}$ de longitud.

Soares et al. (2011) realizaron mediciones en parásitos hallados en 5 eritrocitos y 2 leucocitos, observando formas parasitarias de formas ovales a redondeadas con un núcleo excéntrico y citoplasma levemente basófilo. Si bien es común encontrar células infectadas con un solo parásito, hallaron de 6 a 33 merozoítos en cada leucocito. Las medidas halladas por estos autores fueron fue $2,67 \pm 0,40 \mu \mathrm{m}$ de largo por 1,94 $\pm 0,22 \mu \mathrm{m}$ de ancho. Los núcleos midieron, en promedio, $1,06 \pm 0,17 \mu \mathrm{m}$ de largo por $0,89 \pm 0,15$ $\mu \mathrm{m}$ de ancho.

En este trabajo se observaron diferencias morfológicas y morfométricas entre los parásitos hallados en leucocitos y en eritrocitos. En monocitos fue frecuente encontrar vacuolas conteniendo varios parásitos agrupados, de manera similar a los descriptos previamente en células endoteliales, aunque también se hallaron libres en el citosol. En neutrófilos, solo se observaron merozoítos libres en el citosol. Estos hallazgos podrían implicar alguna diferencia en cuanto al estadio del parásito. Podría considerarse que los merozoítos tipo 1 corresponden a etapas en activa división, dada la frecuencia en la que se encuentran estructuras compatibles con división nuclear. Sin embargo, todavía falta conocer completamente el ciclo parasitario en infecciones naturales para poder aceptar estas hipótesis. Para algunos autores, la presencia de formas libres en plasma sería el resultado de la fragilidad de las células durante el manejo de la muestra, ya que los piroplasmas son considerados parásitos intracelulares y estarían más relacionados con un artefacto que con una verdadera etapa parasitaria (Loretti \& Barros, 2005).

Morfológicamente, los merozoítos tipo 1 de $R$. vitalii podrían ser confundidos con amastigotes de Leishmania spp., que poseen un tamaño similar con forma oval o redondeada. Más aún, la zona geográfica de distribución de rangeliosis en nuestro país coincide con la zona endémica de leishmaniosis canina. Sin embargo, es muy infrecuente el hallazgo de amastigotes en las células sanguíneas y, además, en ellos se destaca la presencia de cinetoplasto.

Este trabajo aporta datos morfológicos y morfométricos de utilidad en el diagnóstico parasitológico de rangeliosis canina.

\section{Conflicto de intereses}

Todos los autores declaran que no existe conflicto de intereses, incluyendo entre estos últimos las 
relaciones financieras, personales o de otro tipo con otras personas $u$ organizaciones que pudieran influir de manera inapropiada en el trabajo.

\section{Bibliografía}

Carini A, Maciel J. 1914. Sobre a molestia dos caes, chamada Nambi-Uvú, e o seu parasita (Rangelia vitalii). Annaes Paulistas de Medicina e Cirugia 3:65-71.

Colombo VC, Pereira J, Nava S, Beldomenico PM. 2016. Amblyomma aureolatum (Acari: Ixodidae): nuevos registros en las provincias de Buenos Aires y Santa Fe, Argentina. Revista FAVE - Sección Ciencias Veterinarias. 15:1-4. doi: 10.14409/favecv.v15i1/2.5914.

Da Silva AS, Franca RT, Costa MM, Paim CB, Paim FC, Dornelles GL, Soares JF, Labruna MB, Mazzanti CM, Monteiro SG, Lopes ST. 2011. Experimental infection with Rangelia vitalii in dogs: acute phase, parasitemia, biological cycle, clinical-pathological aspects and treatment. Experimental Parasitology. 128:347-52. doi: 10.1016/j.exppara.2011.04.010.

Da Silva AS, Martins DB, Soares JF, Franca RT. 2012. Canine rangeliosis: the need for differential diagnosis. Parasitology Research. 112:1329-32. doi:10.1007/s00436-012-3160-y.

Eiras DF, Craviotto MB, Baneth G, Moré G. 2014. First report of Rangelia vitalii infection (canine rangeliosis) in Argentina. Parasitology International. 63:729-34.

doi: 10.1016/j.parint.2014.06.003.

Fighera RA, Souza TM, Kommers GD, Irigoyen LF, Barros CSL. 2010. Patogênese e achados clínicos, hematológicos e anatomopatológicos da infeccão por Rangelia vitalii em 35 cães (1985-2009). Pesquisa Veterinaria Brasilera. 30:97487. doi10.1590/S0100-736X2010001100012.

Franca RT, Da Silva AS, Paim FC, Costa MM, Soares JF, Mazzanti CM, Lopes STA. 2010. Rangelia vitalii in dogs in southern Brazil. Comparative Clinical Pathology. 19:383-87. doi:10.1007/s00580-010-1041-2.

Franca RT, Da Silva AS, Loretti AP, Mazzanti CM, Lopes STA. 2014. Canine rangeliosis due to Rangelia vitalii: From first report in Brazil in 1910 to current day - A review. Ticks and tickborne diseases. 5:466-74. doi 10.1016/j.ttbdis.2014.04.005

Fredo G, Bianchi M, De Andrade C, De Souza S, Leite-Filho R, Bandinelli M, Amorim DB, Driemeier D, Sonne L. 2015. Natural infection of wild canids (Cerdocyon thous and Lycalopex gymnocercus) with the intraendothelial piroplasm Rangelia vitalii in Southern Brazil. Journal of Wildlife Diseases. 51:880-4. doi: 10.7589/2014-12-283.

Guglielmone AA, Mangold AJ, Boero CA, Azcue RA. 2002. Nuevo registro de Amblyomma aureolatum (Pallas, 1772) (Amblyomma striatum Koch, 1884) en Entre Ríos, Argentina. Revista FAVE - Ciencias Veterinarias. 1(2):33-5.

Loretti AP, Barros SS. 2005. Hemorrhagic disease in dogs infected with an unclassified intraendothelial piroplasm in southern Brazil. Veterinary Parasitology. 134:193-213. doi:10.1016/j.vetpar.2005.07.011.

Pestana BR. 1910. O Nambyuvú. Rev Méd São Paulo 22:423-6.

Sanchez RO, Moré G, Eiras DF. 2014. Piroplasmosis canina por Rangelia vitalii (Protozoa, Piroplasmida) en la ciudad de Concordia, Entre Ríos. XX Reunión Científico Técnica de la AAVLD. Tucumán, Argentina.
Sarasúa LM, Donati NR. 1976. Constatación de babesiosis canina en el Dpto. de Artigas (Uruguay). Veterinaria (Montevideo) 62:137-9.

Soares JF, Girotto A, Brandão PE, França, RT, Da Silva AS, Lopes STA, Labruna M. 2011. Detection and molecular characterization of a canine piroplasm from Brazil. Veterinary $\mathrm{Pa}-$ rasitology. 180(3-4):203-8. doi:10.1016/j.vetpar.2011.03.024.

Soares JF. 2014. História natural da rangeliose. Tesis de doctorado en Ciencias Veterinarias, Faculdade de Medicina Veterinária e Zootecnia, Universidade de São Paulo. doi: 10.11606/T.10.2014.tde-19092014-092438.

Soares JF, Carvalho L, Maya L, Dutra F, Venzal JM, Labruna MB. Molecular detection of Rangelia vitalii in domestic dogs from Uruguay. Veterinary Parasitology. 2015; 210(1-2):98101. doi:10.1016/j.vetpar.2015.03.013. 